\title{
Trajectory versus probability density entropy
}

\author{
Mauro Bologna ${ }^{1}$, Paolo Grigolini ${ }^{1,2,3}$, Markos Karagiorgis ${ }^{1}$ \\ ${ }^{1}$ Center for Nonlinear Science, University of North Texas, \\ P.O. Box 5368, Denton, Texas 76203 \\ ${ }^{2}$ Istituto di Biofisica del Consiglio Nazionale delle \\ Ricerche, Via San Lorenzo 26, 56127 Pisa, Italy \\ ${ }^{3}$ Dipartimento di Fisica dell'Università di Pisa, \\ Piazza \\ Torricelli 2, 56127 Pisa, Italy
}

(November 9, 2018)

\begin{abstract}
We study the problem of entropy increase of the Bernoulli-shift map without recourse to the concept of trajectory and we discuss whether, and under which conditions if it does, the distribution density entropy coincides with the Kolmogorov-Sinai entropy, namely, with the trajectory entropy.

05.45.+b,03.65.Sq,05.20.-y
\end{abstract}

Typeset using REVTEX 


\section{INTRODUCTION}

The problem of establishing a connection between the Kolmogorov-Sinai (KS) entropy [1:2] and the conventional entropy expressed in terms of probability density is an interesting problem that is attracting some attention in literature [3, 4 . Early work on this subject goes back to the discussion of Goldstein and Penrose [5]: These authors, almost twenty years ago, established a connection between the KS entropy and a coarse-grained version of the distribution density entropy. The work of Ref. [5] is based on a formal and rigorous mathematical treatment which for this reason might have eluded the attention of physicists working on this subject [3, [4]. Thus we restate the problem using intuitive arguments which also make it possible for us to account for the more recent literature on the subject. In fact, our heuristic treatment will allow us to relate the results of the more recent work of Latora and Baranger [四] to the earlier work of Zurek and Paz [3].

In addition to revisiting the problem of how to make the $\mathrm{KS}$ entropy emerge from a nonequilibrium dynamic picture [5], we shall touch also the intriguing problem of whether a thermodynamic perspective has to rest on the adoption of trajectories, as implied by the concept itself of KS entropy, or on the use of probability densities, advocated with strong arguments by Petrosky and Prigogine [6,7]. It is convenient to stress that the KS entropy [1]:2] is a property of a single trajectory. The phase space is divided into cells, each cell being assigned a given label $\omega_{r}$. Then we define a sequence of symbols by means of a single trajectory: The sequence is determined assigning to any time step the label of the cell where the trajectory lies at that time step. The trajectory is supposed to be large enough as to yield reliable values for the probabilities determined through the numerical frequencies. This means that we fix a window of size $N$, and we move this window along the sequence. For

any window position a string of symbols $\omega_{0}, \omega_{1}, \ldots \omega_{N-1}$ is determined. Moving the window of fixed size $N$ along the infinite sequence generated by the trajectory we have to evaluate how many times the same string of symbols appears, thereby leading us to determine the probability $p\left(\omega_{0}, \omega_{1}, \ldots \omega_{N-1}\right)$. The KS entropy is then defined by 


$$
h_{K S} \equiv \lim _{N \rightarrow \infty} H(N) / N
$$

where $H(N)$ is the conventional Shannon entropy of the window of size $N$ defined by

$$
H(N)=\sum_{\omega_{0}, \omega_{1} \ldots \omega_{N-1}} p\left(\omega_{0}, \omega_{1}, \ldots \omega_{N-1}\right) \ln \left[p\left(\omega_{0}, \omega_{1}, \ldots \omega_{N-1}\right)\right] .
$$

It is evident therefore that the KS entropy rests on trajectories, and, more specifically, it implies the adoption of only one trajectory of virtually infinite length. The KS entropy is very attractive because its value turns out to be independent of the repartition into cells of the phase space, due to the crucial role of the so called generating partitions $[\mathbb{8}]$. In the specific case where a natural invariant distribution exists, it is shown [9] that

$$
h_{K S}=\sum_{i} \int d \mathbf{x} \rho_{e q}(\mathbf{x}) \lambda_{i}(\mathbf{x})
$$

with $\lambda_{i}(\mathbf{x})>0$.Note that $\mathbf{x}$ denotes the coordinate of a multidimensional phase space, $\rho_{e q}(\mathbf{x})$ is the natural invariant distribution and $\lambda_{i}(x)$ is a local Lyapounov coefficient, with $i=1, d, d$ being the dimension of the system under study. From Eq.(3) we see that, as earlier pointed out, the KS entropy is independent of the repartition into cells. The original definition of Eq.(11), with $N$ thought of as time, means that the KS entropy, as a property of a single trajectory, is the rate of entropy increase per unit of time. However, since the single trajectory under examination is infinitely long, and explores in time all the phase space available, the KS entropy can also be expressed in the form of an average over the equilibrium distribution density, without any prejudice for the single trajectory nature of this "thermodynamic" property.

According to Petrosky and Prigogine [6, [], on the contrary, the connection between dynamics and thermodynamics implies the use of the Liouville equation

$$
\frac{\partial}{\partial t} \rho(\mathbf{x}, t)=-i L \rho(\mathbf{x}, t)
$$

where $L$ denotes both the classical and the quantum Liouville operator, and $\rho(\mathbf{x}, t)$ is the nonequilibrium distribution density. The reason for this choice is that the analysis of the Liouville operator, through the "rigged Hilbert" space, allows the appearance of complex 
eigenvalues which correspond to irreversibility, and to the collapse of trajectories as well. This is the reason why distribution densities are judged to be more fundamental than trajectories.

In this paper we limit our analysis to the special case where dynamics are generated by maps rather than by Hamiltonians. We do not address the difficult issue of discussing the thermodynamic limit $N \rightarrow \infty$ which is the subject of very interesting recent discussions [7.10], and where, according to Lebowitz [10], ergodicity and mixing are neither necessary nor sufficient to guarantee the connection between dynamics and thermodynamics. We consider the case of low-dimension chaos, where probability emerges as a consequence of sensitivity to initial conditions [7]. Even in this case, however, according to the perspective established by Petrosky and Prigogine [6,7], probability densities are more fundamental than trajectories. The readers interested in knowing more about this perspective, entirely based on probability density, should consult the illuminating work of Driebe [11]. In this case the counterpart of Eq.(四) becomes

$$
\rho(\mathbf{x}, t+1)=\Lambda \rho(\mathbf{x}, t)
$$

where $\Lambda$ is referred to as Frobenius-Perron operator. Of course, the operator $L$ of Eq.(化) has to be identified with $i(\Lambda-1)$.

According to the traditional wisdom, the Frobenius-Perron operator is expected to make the distribution densities evolve in the same way as that resulting from the time evolution of a set of trajectories with initial conditions determined by the initial distribution density |11]: The known cases of discrepancy between the two pictures are judged to be more apparent than real [12]. Nevertheless, even in the case of invertible maps, the birth of irreversibility can be studied using the same perspective as that adopted for Hamiltonian systems, with Eq.(14) replaced by Eq.(5), and so using again probability densities rather than trajectories.

However, we attempt at digging out the KS entropy from Eq.(5), and this purpose forces us to formulate a conjecture on how to relate entropy to $\rho(\mathbf{x}, t)$. A plausible choice seems to be 


$$
S(t)=-\int_{\mathbf{X}} \rho(\mathbf{x}, t) \ln [\rho(\mathbf{x}, t)] d \mathbf{x}
$$

We share the view of Goldstein and Penrose [5] who consider the KS entropy to be a nonequilibrium entropy. In other words, we may hope to derive the KS entropy from the time derivative of $S(t)$ of Eq.(6). As Goldstein and Penrose do [5], to realize that purpose we have to address a delicate problem: In the case of invertible maps, $S(t)$ is time independent [13], thereby implying a vanishing KS entropy. Yet, the baker's transformation, which is a well known example of invertible map, thereby yielding a time independent $S(t)$, is shown 14 to yield a KS entropy equal to $\ln 2$, a fact suggesting a steady condition of entropy increase. We plan to discuss all this with the joint use of heuristic arguments and of the rigorous theoretical tools of Ref. [11.

The present paper uses as a paradygm of invertible map the two-dimensional baker's transformation, depending on two coordinates, $x$ and $y$, the former corresponding to dilatation and the latter to contraction. Using this prototype for invertible dynamics, we aim at proving that the adoption of the distribution density in the case of invertible chaotic maps would lead to an increasing process of fragmentation, depending not only, as the KS entropy does, on the positive Lyapounov coefficient, but also on the negative one. The adoption of a coarse graining has the effect of quenching the action of the negative Lyapunov coefficient, thereby allowing the KS entropy to show up. Then, to go beyond these heuristic arguments we make a trace on the variable $y$, namely, on the process responsible for contraction, and we focus our attention on the contracted dynamics. This is equivalent to that produced by the Bernoulli shift map. Here room is only left for dilatation and the problem can be solved with a rigorous mathematical method, without using trajectories.

The outline of the paper is as follows. In Section II we shall illustrate our heuristic picture. In Section III we shall address the problem by means of a rigorous treatment resting on the theoretical tools provided by Driebe [11]. In Section IV we shall draw some conclusions. Some delicate mathematical problems behind the theoretical calculations of Section III are detailed in Appendix. 


\section{HEURISTIC ARGUMENTS}

Note that the cases studied by Latora and Baranger [4] are two-dimensional, and our discussion here refers to a two-dimensional case, too. We have in mind the backer's transformation and $\mathbf{x} \equiv(x, y)$. We denote by $W(t)$ the number of cells occupied at a given time $t$. Note that $W(0)<W_{\max }$, where the symbol $W_{\max }$ denotes the total number of cells into which we have divided the phase space $\mathbf{X}$. Our heuristic approach is based on the following assumptions.

(i) At the initial time only $W(0)$ cells are occupied.

(ii) At all times the trajectories are equally distributed over the set of occupied cells. This means

$$
S(t)=\ln W(t) .
$$

(iii) We denote by $\lambda$ the positive Lyapounov coefficient, and we set

$$
W(t)=W(0) \exp (\lambda t)
$$

All these three assumptions have been borrowed from the recent work of Ref. [15]. The joint use of all them yields

$$
S(t)=\lambda t-\ln W(0),
$$

which corresponds to the Kolmogorov thermodynamical regime. Note that the positive Lyapounov coefficient in the case of the baker's transformation is shown [14] to be:

$$
\lambda=\ln 2 .
$$

Note also that according to the arguments of Section I, the connection with the KS entropy is established through the time derivative of $S(t)$. Thus, we conclude that

$$
\frac{d S}{d t}=\lambda=\ln 2=h_{K S},
$$

which corresponds to deriving the KS entropy from the distribution density picture. 
This Kolmogorov regime is not infinitely extended. It has an upper bound, given by the fact that when equilibrium is reached, even in the merely sense of a coarse-grained equilibrium, then the entropy stops increasing. An estimate of this time is obviously given by the solution of the following equation

$$
\ln W_{\max }=\lambda t-\ln W(0),
$$

which yields the following saturation time

$$
t_{S}=\frac{1}{\lambda} \ln \left(\frac{W_{\max }}{W(0)}\right) .
$$

Furthermore a lower bound of validity exists, which will be easily estimated with very simple arguments. If the initial distribution includes a large number of cells and the size of this distribution along the coordinate $y$ is $L$, and the size of the cells is $\epsilon$ with $\epsilon<L$, then it is evident that, in spite of the coarse graining the total number of cells occupied remains the same for a while. This time is easily estimated using the equation

$$
\operatorname{Lexp}(-\lambda t)=\epsilon
$$

which in fact defines the time at which the distribution volume, and consequently, the system entropy starts increasing. This time is denoted by the symbol $t_{D}$ and reads

$$
t_{D}=\frac{1}{\lambda} \ln \left(\frac{L}{\epsilon}\right)
$$

We denote by $U(t)$ the volume of the distribution density at time $t$ and by $V$ the volume of the phase space, thereby implying that $U(t) \leq V$. We note that

$$
\frac{W_{\max }}{W(0)}=\frac{V}{U(0)},
$$

where $V$ is the total volume of the phase space and $U(0)$ is the initial volume of the distribution density. Thus the Kolmogorov regime shows up in the following time interval

$$
t_{D}=\frac{1}{\lambda} \ln \left(\frac{L}{\epsilon}\right)<t<t_{S}=\frac{1}{\lambda} \ln \left(\frac{V}{U(0)}\right) .
$$


The time duration of the regime of validity of the Kolmogorov regime can be made infinitely extended by making the cell size infinitely small. This means that the conflict between the KS entropy prescription and the time independence of $S(t)$ can be bypassed by focusing our attention on the intermediate region, whose time duration tends to infinity with $\epsilon \rightarrow 0$. We note that a choice can be made such that $V / U(0)=(L / \epsilon)^{\chi}$, with $\chi>1$. This means the time duration of the Kolmogorov regime can be made $\chi$ times larger than the time duration of the transition regime. For $\epsilon \rightarrow 0$ both time durations become infinite, thereby showing that a Kolmogorov regime of infinite time duration can be obtained at the price, however, of waiting an infinitely long time for the entropy to increase. The infinite waiting time before the regime of entropy increase fits the observation [13,12 that the Gibbs entropy of an invertible map is constant. The linear entropy increase showing up "after this infinite waiting time" allows the emergence of the KS entropy from within the probability density perspective.

This kind of coarse graining might be criticized as corresponding to arbitrary choices of the observer. It is interesting to remark that there exists another interesting form of coarse graining, produced by weak stochastic forces. Both in the case where this stochastic forces mimic the interaction with the environment [3] or in the case where it happens to be an expression of spontaneous fluctuations [16] this kind of coarse graining can be regarded as being produced by nature. Here we limit ourselves to remarking that according to Zurek and Paz [3] these stochastic forces contribute a fluctuation-dissipation process mimicking the interaction between the system of interest and the environment. These authors studied the inverted stochastic oscillator

$$
\frac{d^{2} x}{d t^{2}}=\lambda^{2} x(t)+\gamma \frac{d x}{d t}+f(t)
$$

where the friction $\gamma$ and the stochastic force $f(t)$ are related to one another by the standard fluctuation-dissipation relation

$$
<f f(t)>=2 \gamma<\left(\frac{d x}{d t}\right)^{2}>_{e q} \delta(t) \equiv 2 D \delta(t)
$$


It is interesting to remark that the proper formulation of the second principle implies that the entropy of a system can only increase or remain constant under the condition of no energy exchange between the system and its environment. In the case of Eq.(19) the energy exchange between system and environment is negligible for any observation made in the time scale

$$
t<<1 / \gamma
$$

To ensure that the system entropy increase to take place with no energy exchange between system and its environment Zurek and Paz [3] set the condition of Eq.(20) and this, in turn, allows them to neglect the friction term in Eq.(18). Then, these authors adopted the modes

$$
u \equiv \frac{d x}{d t}+\lambda x
$$

and

$$
w \equiv \frac{d x}{d t}+\lambda x
$$

which make it possible for them to split Eq.(18) into

$$
\frac{d u}{d t}=\lambda u(t)+f(t)
$$

and

$$
\frac{d w}{d t}=-\lambda w(t)+f(t)
$$

Let us imagine the initial distribution density as a rectangle of size $\Delta w(0)$ along the direction $w$ and $\Delta u(0)$ along the direction $u$. We keep denoting by $U(t)$ the distribution volume at a given time $t$. Thus the volume of the initial distribution is

$$
U(0)=\Delta u(0) \Delta w(0)
$$

In the absence of the stochastic force $f(t)$, Eqs.(23) and Eqs.(23) result in an exponential increase and an exponential decrease, with the same rate $\lambda$, respectively. Consequently, the 
Liouville theorem $U(t)=U(0)$ is fulfiled. In the presence of stochastic force, we work as follows. In the former equation, with $u$ increasing beyond any limit, the weak stochastic force $f(t)$ can be neglected. This is not the case with the latter equation. In fact, $w$ is a contracting variable in the absence of the stochastic force. In the presence of the stochastic force the minimum size of the distribution along $w$ is given by

$$
<w^{2}>_{e q}^{1 / 2}=(D / \lambda)^{1 / 2}
$$

This minimum size is reached in a time determined by the solution of the following equation

$$
\Delta w(0) \exp (-\lambda t)=(D / \lambda)^{1 / 2}
$$

yielding

$$
t_{D}=\frac{1}{\lambda} \ln \left(\frac{\lambda}{D}\right)^{1 / 2} \Delta w(0)
$$

Due to the fact that deterministic chaos is simulated by Zurek and Paz [3] by means of an inverted parabola, these authors did not consider the entropy saturation effects. However, it is straigthforward to evaluate the saturation effect with heuristic arguments concerning the case where the total volume of the phase space has the finite value $V$. From the time $t=t_{D}$ on, the distribution volume $U(t)$ increases exponentially in time with the following expression

$$
U(t)=\Delta w(0) \Delta u(0) \exp (\lambda t)=(D / \lambda)^{1 / 2} \Delta u\left(t_{D}\right) \exp (\lambda t)
$$

Thus, the saturation time is now given by

$$
t_{S}=\frac{1}{\lambda} \ln \left[\frac{V}{\Delta u(0) \Delta w(0)}\right] .
$$

Using Eq.(25) we can write this saturation time as

$$
t_{S}=\frac{1}{\lambda} \ln \left[\frac{V}{U(0)}\right]
$$

which coincides with Eqs.(17) and (13). 
In conclusion, it seems that the emergence of a Kolmogorov regime is made possible by the existence of a form of coarse graining, and that it is independent of whether the coarse graining is realized by the division into cells or by a weak stochastic force. This property seems to make less important the discussion of whether the stochastic force is of environmental origin or rests on some kind of extension of the current physical laws. However, we have to point out that the situation significantly changes, if we move from a strongly to a weakly chaotic classical system. As a relevant example, let us refer ourselves to the work of Ref. [17]. The authors of this work study the asymptotic time limit of a diffusion process generated by using an intermittent map as a dynamic generator of diffusion. If these dynamics are perturbed by a white noise, a transition is provoked, at long times, from anomalous to normal diffusion. When the only source of random behavior is given by the sporadic randomness of the intermittent map [18], the long-time limit is characterized by Lévy statistics, a physical condition in a striking conflict with the condition of Gaussian statistics produced by the action of fluctuations [17]. Here we limit our attention to the case of strong chaos where the two distinct sources of coarse graining produce equivalent effects. It might be of some interest for the reader to compare the coarse-graining approach of this section to the more formal method recently adopted by Fox [19] to deal with the same problem.

It is interesting to stress that to make the regime of validity of the Kolmogorov regime as extended as possible we must make the ratio $V / U(0)$ as large as possible (virtually infinite). This means that we have to choose an initial distribution density so sharp as to become apparently equivalent to a single trajectory. This seems to be an attractive way of explaining why in this condition the KS entropy is recovered, since, as stressed in Section I, the KS entropy is a single trajectory property. However, in accordance with the authors of Refs. [6,7,11] we must admit that there exists a deep difference between a trajectory and a very sharp distribution. The latter is stable and robust, while the former is not. In Section III we shall show that the rigorous derivation of the Kolmogorov regime requires a non trivial mathematical procedure, and the mathematical effort to make from this side, to derive the 
KS entropy, serves the useful purpose of proving that the KS entropy of a trajectory is a really wise way of converting into advantages the drawbacks of the trajectory instability.

\section{THE KS ENTROPY FROM A REDUCED FROBENIUS-PERRON EQUATION}

This Section is devoted to a rigorous discussion resting only on the theoretical tools described in Ref. [11] for a genuine probability density aproach. According to Mackey [13], if we rule out the possibility that the laws of physics are misrepresented by invertible dynamic

prescriptions, there are only two possible sources of entropy increase. The first is the coarse graining discussed in Section II. The second is the adoption of reduced equation of motion, obtained by a trace over "irrelevant" degrees of freedom [13. In fact here we study the Bernoulli shift map,

$$
x_{t+1}=2 x_{t}, \bmod 1 \text {. }
$$

The Frobenius-Perron equation of this map is defined by [11]

$$
\rho(x, t+1)=\Lambda \rho(x, t) \equiv \frac{1}{2}\left[\rho\left(\frac{x}{2}, t\right)+\rho\left(\frac{x+1}{2}, t\right)\right] .
$$

It is straigtforward to show that the Frobenius-Perron operator of Eq.(33) stems from the contraction over the variable $y$ of the baker's mapping, acting in fact on the unit square of two-dimensional space $(\mathrm{x}, \mathrm{y})$ (see, for instance Ref. [14). It is shown 14 that the KS entropy of the baker's transformation is well defined and turns out to be the same as that of the Bernoulli shift map, namely $h_{K S}=\ln 2$. Intuitively, this suggests that the main role of coarse graining is that of making inactive the process of contraction, and with it the negative Lyapunov coeficient. This intuitive argument seems to be plausible and raises the interesting question of how to prove it with a rigorous approach. This is equivalent to deriving the Kolmogorov regime using a rigorous mathematical method rather than the heuristic arguments of Section II. We must observe again that this is made possible by the fact that the tracing has changed the originally invertible map into one that is not invertible. 
To address this issue we follow the prescription of Ref. [11]. First of all, we express the distribution density at time $t$ under the form given by Ref. [11] which reads:

$$
\rho(x, t)=1+\sum_{j=1}^{\infty} \exp \left(-\gamma_{j} t\right) \frac{B_{j}(x)}{j !}\left[\rho^{(j-1)}(1,0)-\rho^{(j-1)}(0,0)\right] .
$$

Note that $\gamma_{j} \equiv j \ln 2, B_{j}(x)$ are the Bernoulli polynomials [20] and $\rho^{(n)}(x, t)$ denotes the $n$-th order derivative of $\rho(x, t)$ with respect to $x$. Hereby, we shall show how to derive from the previous one more tractable expression, which will be checked in appendix.

In the case of an initial condition close to equilibrium, resulting from the sum of the equilibrium distribution and the first "excited" state, it is easy to prove that the entropy $S(t)$ of Eq. (6) reaches exponentially in time the steady-state condition. This suggests that the Kolmogorov regime, where the entropy $S(t)$ is expected to be a linear function of time, must imply an initial condition with infinitely many "excited" states. To deal with a condition of this kind it is convenient to express Eq.(34) in an equivalent form given by

$$
\rho(x, t)=1+\sum_{j=1}^{\infty} \int_{-\infty}^{+\infty} \frac{B_{j}(x)}{j !}(-i z \omega)^{j-1} \hat{\rho}(\omega)(\exp (-i \omega)-1) \frac{d \omega}{2 \pi}
$$

where $z \equiv \exp [-t(\ln 2)]$ and $\hat{\rho}(\omega)$ is related to the initial condition $\rho(x, 0)$ by the Fourier transform

$$
\rho(x, 0)=\int_{-\infty}^{+\infty} \hat{\rho}(\omega) \exp (-i \omega x) \frac{d \omega}{2 \pi}
$$

The following equation

$$
\sum_{j=0}^{\infty} \frac{B_{j}(x)}{j !} z^{j}=z \frac{\exp (z x)}{\exp (z)-1}
$$

is known [20] to generate Bernoulli polynomials. Using this Bernoulli polynomial generatrix, we arrive, after some algebra, at

$$
\rho(x, t)=z \int_{-\infty}^{+\infty} \exp (-i \omega z x) \hat{\rho}(\omega) \frac{\exp (-i \omega)-1}{\exp (-i \omega z)-1} \frac{d \omega}{2 \pi}
$$

By expanding the denominator of Eq.(38) into a Taylor series and using Eq.(36), we finally derive the fundamental expression 


$$
\rho(x, t)=z \sum_{n=0}^{\infty}[\rho(z x+z n, 0)-\rho(z x+z n+1,0)] .
$$

This important expression makes it possible for us to discuss analytically the entropy time evolution ensuing the preparation of an initially very sharp distribution. Let us consider in fact

$$
\rho(x, 0)=\frac{\alpha}{1-\exp (-\alpha)} \exp (-\alpha x), 0 \leq x \leq 1 .
$$

For $\alpha \rightarrow \infty$ this initial distribution becomes a very sharp distribution located at $x=0$. By plugging this initial distribution density into Eq.(39) we obtain

$$
\rho(x, t)=z \alpha \frac{\exp (-\alpha x z)}{1-\exp (-\alpha z)}
$$

It is evident that this simple analytical expression for the time evolution of the distribution density is exact, and corresponds to the time evolution dictated by the Frobenius-Perron operator of Eq. (33).

We are now in a position to discuss the central issue of this paper, namely, the time evolution of the Gibbs entropy of Eq.([6), which, in the case here under study, reads

$$
S(t)=-\int_{X} \rho(x, t) \ln [\rho(x, t)] d x
$$

with $X$ now denoting the interval [0,1]. By plugging Eq.(40) within Eq.(42) we obtain

$$
S(t)=1-\ln (\alpha z)+\ln [1-\exp (-\alpha z)]-\frac{\alpha z}{\exp (\alpha z)-1}
$$

In the limiting case $\alpha \rightarrow \infty$ this exact prediction is approximated very well by

$$
S(t)=-\ln (\alpha)+(\ln 2) t
$$

It indicates that a sharp initial distribution makes the system evolve according to the KS entropy, with no regime of transition from mechanics to thermodynamics. The third regime of Ref. [4] is still present. It is straigtforward to show that the saturation time $t_{S}=\ln \alpha / \ln 2$ resulting from Eq.(43) is the same as that of Eq.(13) in the case $V=1$. In fact using 
Eq.(16) and $V=1$ we obtain that $W_{\max } / W(0)=1 / U(0)$, where $U(0)$ is the size of the initial distribution. The size of the initial distribution of Eq.(40), for $\alpha \rightarrow \infty$, becomes prportional to $1 / \alpha$. Thus, $\ln \alpha \approx \ln \left(W_{\max } / W(0)\right.$ in accordance with Eq. (13).

This is an elegant result, involving a modest amount of algebra. However, it refers to an initial distribution located at $x=0$. We want to prove that this is a general property, independent of where the initially sharp distribution is located, at the price, as we shall see, of a more complicated mathematical treatment. For this purpose we study the case where the distribution shape is the Lorentzian curve:

$$
\rho(x, 0)=A \frac{\Gamma}{\left(x-x_{0}\right)^{2}+\Gamma^{2}},
$$

with $x_{0}$ being a generic point of the interval $[0,1]$ and $x$ running in the same interval. Setting the normalization condition yields

$$
A=\frac{1}{\arctan \left(\frac{x_{0}}{\Gamma}\right)+\arctan \left(\frac{1-x_{0}}{\Gamma}\right)} .
$$

We have to set again the condition that the initial distribution is very sharp. Thus we make the assumption $\Gamma \rightarrow 0$, yielding $A \approx 1 / \pi$. We plug this approximated value of $A$ into Eq. (39), thereby obtaining the following density time evolution

$$
\rho(x, t)=\frac{z \Gamma}{\pi} \sum_{n=0}^{\infty}\left[\frac{1}{\left(z x+z n-x_{0}\right)^{2}+\Gamma^{2}}-\frac{1}{\left(z x+z n-x_{0}+1\right)^{2}+\Gamma^{2}}\right] .
$$

We are now in a position to study the entropy time evolution again. Plugging Eq.(47) into (42) we find

$$
\begin{array}{r}
S(t)=-\int_{X} \rho(x, t) \ln \left[\frac{z}{\pi \Gamma} \sum_{n=0}^{\infty} \frac{1}{\left(\frac{z x+z n-x_{0}}{\Gamma}\right)^{2}+1}-\frac{1}{\left(\frac{z x+z n-x_{0}+1}{\Gamma}\right)^{2}+1}\right] d x \\
=-\int_{X} \rho(x, t) \ln \left[\frac{z}{\pi \Gamma}\right] d x-\int_{X} \rho(x, t) \ln \left[\sum_{n=0}^{\infty} \frac{1}{\left(\frac{z x+z n-x_{0}}{\Gamma}\right)^{2}+1}-\frac{1}{\left(\frac{z x+z n-x_{0}+1}{\Gamma}\right)^{2}+1}\right] d x,
\end{array}
$$

where $[y]$ denotes the integer part of $y$. To derive a more tractable expression we note that in the limiting case of $\Gamma$ very small, the quantities [...] contributing this series are almost 
zero except for $n=-[x]+\left[\frac{x_{0}}{z}\right]=\left[\frac{x_{0}}{z}\right]$ in the first term, and for the possible contribution

$n=-[x]-\left[\frac{1-x_{0}}{z}\right]=-\left[\frac{1-x_{0}}{z}\right]$ in the second term. The latter condition cannot be realized, since $n$ is a positive integer. Thus, using only the first class of contributions, we get for the entropy $S(t)$ the following approximate expression

$$
S(t) \approx \ln \Gamma+(\ln 2) t-\ln \pi-\int_{0}^{z / \Gamma} \frac{\ln \left(y^{2}+1\right)}{y^{2}+1} d y
$$

which, in the limiting case $\frac{z}{\Gamma} \rightarrow \infty$ becomes

$S(t) \approx \ln \Gamma+(\ln 2) t-\ln \pi-\int_{0}^{\infty} \frac{\ln \left(y^{2}+1\right)}{y^{2}+1} d y=\ln \Gamma+(\ln 2) t-\ln \pi-\pi \ln 2 \approx \ln \Gamma+(\ln 2) t$.

As in the earlier case, the validity of the approximation yielding the linear dependence of $S(t)$ on time, is broken at the time $t \sim-\ln \Gamma / \ln 2$. In conclusion, the Kolmogorov condition is realized by very sharp initial distributions. Our derivation of this interesting property was done adhering to the recommendation of Ref. [6,6,11] of resting only on densities rather than on trajectories.

\section{CONCLUDING REMARKS}

This paper shows that there exists a subtle difference between a Liouville density and a probability distribution. The Liouville distribution density of a chaotic map, which is at the same time invertible, becomes increasingly fragmented with time. If the initial distribution has a volume which is much smaller than the volume of the phase space, the highly fragmented distribution density at large times has a volume identical to the initial, but the impression afforded by a coarse-grained observation is that the initial volume increases with time till to become equal to that of the whole phase space. If we do the calculation of the time evolution of the Liouville density observing the motion of many trajectories with slightly different initial conditions, we are forced to adopt a coarse-graining procedure, dictated by the need itself of counting how many trajectories are found at a given time in 
a given small region of the phase space. This has the effect of making the Gibbs entropy increase. If, on the contrary, the calculation genuinely rests on the motion of the Liouville density, thereby implying that a quantum-like formalism is adopted, the Gibbs entropy is constant.

On the other hand, the KS entropy is a trajectory property and this, to first sight, might lead us to believe that, being a trajectory property, cannot be recovered from within an approach genuinely resting on the distribution density. The heuristic arguments used in Section II subtly rest on the trajectory properties and thus on the assumption that the two perspectives are equivalent in spite of the warnings of the authors of Refs. [6,0,11]. This is the reason why we judge the theoretical calculations of Section III to be of significant interest. These results have been obtained without having any direct recourse to the trajectory instability and only using the theoretical tools illustrated in Ref. [11], which in fact address the problem of the dynamics of map using the quantum mechanical language of eigenstate and eigenvalue. It has to be pointed out that to establish this rigorous connection between the linear increase of the Gibbs entropy and the KS entropy we need to use infinitely many "excited" states, namely, a condition very far from equilibrium. The adoption of a "reduced" Liouville-like equation has been essential for the success of this enterprise. The Frobenius-Perron operator of the Bernoulli shift map is obtained from the Frobenius-Perron operator of the baker's transformation via contraction over the variable $y$, corresponding to the contraction process. As a consequence, room is only left for dilatation. This is the reason why the heuristic argument of Eq.(8) holds true with no restriction and the use of a method rigorously based on densities lead to the same result. After all, the adoption of the theoretical tools of Ref. [11] as a rigorous way to evaluate the regression to equilibrium, must reflect in a way the trajectory instabilities behind the Pesin theorem of Eq.(3).

In the case where the action of the negative Lyapounov coefficient is quenched by a coarse-graining process, in addition to the saturation and to the Kolmogorov regime, also a short-time regime of transition to thermodynamics appears, so that, as found in Ref. [4] three distinct regimes can be detected, the regime of transition to thermodynamics, 
the Kolmogorov regime, and the saturation regime. Here we show that the three regimes discussed by Latora and Baranger [4] are exhibited also in the case of a coarse graining produced by random fluctuations.

In conclusion, this paper contributes to deepening our understanding of the connection between density and trajectory distribution. The density entropy exhibits a regime of increase linear in time with a rate equivalent to that of the trajectory entropy if the initial distribution is very sharp. It has to stressed that it cannot be infinitely sharp. This would make the distribution density useless, since an infinitely sharp initial distribution would manifest the lack of robustness pointed out by Driebe [11]. Thus, the connection is established using a genuine density. At the same time the accordance between the heuristic approach yielding the derivation of Eq.(44) can be interpreted as a rigorous support of the assumptions made by some some authors [15] to derive the KS entropy from within a probability distribution approach.

We want to remark that we are not aware of earlier work where the Lyapounov coefficient is derived analytically from a probability density approach with no use of heuristic arguments but that of Pattanayak and Brumer [21] which, however, seems to be limited to the study of the transition regime [22].

A problem open to future research concerns the role of coarse-graining. We have seen that in the case of strong chaos studied in this paper there is no essential difference between the coarse graining resulting from the repartition of the phase space into cells and the coarse graining caused by fluctuations. In the case of dynamic systems like the weakly chaotic billiards studied by Zaslavsky [23,24,, which are easily proved to be statistically equivalent to the intermittent maps of Ref. [17], the two different coarse-graining sources result in different physical effects at long times. On the other hand, it is expected 25 that the dynamic process of transition to Lévy statistics is characterized by thermodynamic properties of non-extensive nature [26]. The study of the time evolution of non-extensive entropy under the actions of the two different sources of coarse graining studied in this paper would an interesting program for future research work. 


\section{APPENDIX}

The purpose of this Appendix is to check the main result of Section III. Our theoretical reference on this issues is given by the book of Ref. [11]. We note, however, that Eqs. (41) and Eq.(47) are not directly derived from Eq.(34), which is a theoretical finding of Ref. [11, but they are derived from Eq.(39), wich is the result of a further development of the theory of Ref. [11]. We feel therefore the need of proving that Eq.(39) fits the main requirement of keeping the norm unchanged and of being an exact solution of Frobenius-Perron equation of Eq.(33). To double check our results, we shall prove also that Eqs. (41) and Eq.(47) fit the same property. As a general remark about the content of this appendix we note that a function of the variable $x$, defined only within the finite interval $[0,1]$, admits a treatment based on its Fourier transform if it is thought of as being defined on the whole interval $[-\infty, \infty]$ with vanishing value outside $[0,1]$. Similarly we can define the Fourier series of this function assuming it to be periodically repeated all over the real axis. We shall adopt this approach throughout the whole appendix.

Let us check Eq.(39) first. We note that the argument of the density of Eq.(39) can be arbitrary with the only condition that the variable $x$ is in the interval $[0,1]$. Furthermore, since Eq.(39) is derived from Eq.(38), it is enough for us to prove that Eq.(38) is properly normalized and is a solution of the Frobenius-Perron equation of Eq.(33). In conclusion, we have to check:

$$
\rho(x, t)=z \int_{-\infty}^{+\infty} e^{-i \omega z x} \hat{\rho}(\omega) \frac{e^{-i \omega}-1}{e^{-i \omega z}-1} \frac{d \omega}{2 \pi} .
$$

First we check that this equation is norm conserving or:

$$
\int_{0}^{1} \rho(x, t) d x=1 .
$$

To do so, we integrate Eq. A-1 with respect to the variable $\mathrm{x}$ from $-\infty$ to $+\infty$. Thus we obtain: 


$$
\begin{aligned}
& \int_{0}^{1} z \int_{-\infty}^{+\infty} e^{-i \omega z x} \hat{\rho}(\omega) \frac{e^{-i \omega}-1}{e^{-i \omega z}-1} \frac{d \omega}{2 \pi} d x \\
= & \int_{-\infty}^{+\infty} \frac{e^{-i \omega z}-1}{-\imath \omega} \hat{\rho}(\omega) \frac{e^{-i \omega}-1}{e^{-i \omega z}-1} \frac{d \omega}{2 \pi} .
\end{aligned}
$$

This means the expression

$$
\int_{-\infty}^{+\infty} \frac{e^{-i \omega}-1}{-\imath \omega} \hat{\rho}(\omega) \frac{d \omega}{2 \pi} \equiv \int_{0}^{1} \int_{-\infty}^{+\infty} e^{-i \omega y} \hat{\rho}(\omega) \frac{d \omega}{2 \pi} d y
$$

The integral over $\omega$ is by definition the Fourier transform of $\rho(y, 0)$, yielding thereby

$$
\int_{0}^{1} \rho(x, t) d x=\int_{0}^{1} \rho(y, 0) d y=1 \forall t
$$

due to the fact that the initial condition is assumed to be normalized.

We want now to prove that the distribution density $\rho(x, t)$ of Eq. (A-1) is a solution of the Frobenius-Peron operator of Eq.(33), namely, that:

$$
\rho(x, t+1)=\frac{1}{2}\left[\rho\left(\frac{x}{2}, t\right)+\rho\left(\frac{x+1}{2}, t\right)\right] .
$$

Remembering that $z=2^{-t}$ and $\frac{z}{2}=2^{-t-1}$ we can write Eq.(A-1)as:

$$
\rho(x, t+1)=\frac{z}{2} \int_{-\infty}^{+\infty} e^{-i \omega z x / 2} \hat{\rho}(\omega) \frac{e^{-i \omega}-1}{e^{-i \omega z / 2}-1} \frac{d \omega}{2 \pi} .
$$

Plugging Eq.(A-1) into it, the r.h.s. of Eq. (A-6) becomes:

$$
\begin{gathered}
\frac{1}{2}\left[\rho\left(\frac{x}{2}, t\right)+\rho\left(\frac{x+1}{2}, t\right)\right] \\
=\frac{z}{2}\left[\int_{-\infty}^{+\infty} e^{-i \omega z x / 2} \hat{\rho}(\omega) \frac{e^{-i \omega}-1}{e^{-i \omega z}-1} \frac{d \omega}{2 \pi}+\int_{-\infty}^{+\infty} e^{-i \omega z(x+1) / 2} \hat{\rho}(\omega) \frac{e^{-i \omega}-1}{e^{-i \omega z}-1} \frac{d \omega}{2 \pi}\right]
\end{gathered}
$$

and after a little algebra we get:

$$
\frac{1}{2}\left[\rho\left(\frac{x}{2}, t\right)+\rho\left(\frac{x+1}{2}, t\right)\right]=\frac{z}{2} \int_{-\infty}^{+\infty} e^{-i \omega z x / 2} \hat{\rho}(\omega) \frac{\left[e^{-i \omega}-1\right]\left[e^{-i \omega z x / 2}+1\right]}{e^{-i \omega z}-1} \frac{d \omega}{2 \pi} .
$$

By decomposing the denominator as follows 


$$
\frac{1}{e^{-i \omega z}-1}=\frac{1}{\left[e^{-i \omega z / 2}-1\right]\left[e^{-i \omega z / 2}+1\right]}
$$

and simplifying, we obtain

$$
\frac{1}{2}\left[\rho\left(\frac{x}{2}, t\right)+\rho\left(\frac{x+1}{2}, t\right)\right]=\frac{z}{2} \int_{-\infty}^{+\infty} e^{-i \omega z x / 2} \hat{\rho}(\omega) \frac{e^{-i \omega}-1}{e^{-i \omega z / 2}-1} \frac{d \omega}{2 \pi}
$$

that coincide with Eq.( $(\mathrm{A}-7)$.

To check the property $\rho(x, t) \rightarrow 1$ for $t \rightarrow \infty$ or $z \rightarrow 0$ we use (A-1) and we write:

$$
\begin{gathered}
\rho(x, t) \sim z \int_{-\infty}^{+\infty} \hat{\rho}(\omega) \frac{e^{-i \omega}-1}{-i \omega z} \frac{d \omega}{2 \pi} \\
=\int_{-\infty}^{+\infty} \hat{\rho}(\omega) \frac{e^{-i \omega}-1}{-i \omega} \frac{d \omega}{2 \pi}=\int_{-\infty}^{+\infty} \hat{\rho}(\omega) \int_{0}^{1} e^{-i \omega x} d x \frac{d \omega}{2 \pi}=\int_{0}^{1} \rho(x) d x=1 .
\end{gathered}
$$

Now we shall test directly the Eq.(41) using Eq.(34). Plugging directly the initial distribution:

$$
\rho(x, 0)=\frac{\alpha}{1-e^{-\alpha}} e^{-\alpha x}, 0 \leq x \leq 1
$$

into Eq.(34) we obtain:

$$
\begin{aligned}
& \rho(x, t)=\sum_{j=0}^{\infty} e^{-\gamma_{j} t} \frac{B_{j}(x)}{j !}\left[\rho^{(j-1)}(1,0)-\rho^{(j-1)}(0,0)\right] \\
= & \frac{\alpha}{1-e^{-\alpha}} \sum_{j=0}^{\infty} e^{-\gamma_{j} t} \frac{B_{j}(x)}{j !}\left[\left(-\alpha^{j-1}\right) e^{-\alpha}-\left(-\alpha^{j-1}\right)\right] .
\end{aligned}
$$

Using the Bernoulli polynomials generatrix [20] we get

$$
\rho(x, t)=z \alpha \frac{e^{-\alpha x z}}{1-e^{-\alpha z}} .
$$

that coincides with Eq.(41) obtaned using the formula Eq.(39)

Finally let us to check the norm conservation of Eq.(47). Without any approximation, using the value of $A$ of Eq.(46), and a little algebra, we get:

$$
\begin{gathered}
\int_{0}^{1} \rho(x, t) d x=\left[\arctan \left(\frac{x_{0}}{\Gamma}\right)+\arctan \left(\frac{1-x_{0}}{\Gamma}\right)\right]^{-1} \\
\cdot \int_{0}^{1} z \Gamma \sum_{n=0}^{\infty}\left[\frac{1}{\left(z x+z n-x_{0}\right)^{2}+\Gamma^{2}}-\frac{1}{\left(z x+z n-x_{0}+1\right)^{2}+\Gamma^{2}}\right] d x \\
=\left[\arctan \left(\frac{x_{0}}{\Gamma}\right)+\arctan \left(\frac{1-x_{0}}{\Gamma}\right)\right]^{-1} \cdot \sum_{n=0}^{+\infty}\left[\arctan \left(\frac{x z+n z-x_{0}}{\Gamma}\right)-\arctan \left(\frac{x z+n z+1-x_{0}}{\Gamma}\right)\right]_{x=0}^{x=1} .
\end{gathered}
$$


This yields:

$$
\begin{gathered}
\int_{0}^{1} \rho(x, t) d x=\left[\arctan \left(\frac{x_{0}}{\Gamma}\right)+\arctan \left(\frac{1-x_{0}}{\Gamma}\right)\right]^{-1} \\
\cdot \sum_{n=0}^{+\infty}\left[\arctan \left(\frac{z+n z-x_{0}}{\Gamma}\right)-\arctan \left(\frac{z+n z+1-x_{0}}{\Gamma}\right)-\arctan \left(\frac{n z-x_{0}}{\Gamma}\right)+\arctan \left(\frac{n z+1-x_{0}}{\Gamma}\right)\right] .
\end{gathered}
$$

Examining the expression we can note that only the terms with $n=0$ survive in the sum but that terms simplify with the external factor (the constant $A$ ) so finally

$$
\int_{0}^{1} \rho(x, t) d x=1 \forall t
$$

Checking that Eq.(47) fulfils the Frobenius-Perron operator involves some extended but straightoforward algebra. 


\section{REFERENCES}

[1] A.N.Kolmogorov,Dokl.Acad.Sci.USSR 119(5), 861 (1958)

[2] Ya.G. Sinai,Dokl.Acad.Sci.USSR 124(4),768 (1959).

[3] W.H. Zurek, and J.P. Paz, Phys.Rev.Lett. 72, 2508 (1994).

[4] V. Latora and M. Baranger, Phys. Rev.Lett. 82, 520 (1999).

[5] S. Goldstein and O. Penrose, J. Stat. Phys. 24, 325 (1981)

[6] T. Petrosky and I. Prigogine, Chaos, Soliton \& Fractals 7,441 (1996).

[7] T. Petrosky and I. Prigogine, Chaos, Soliton \& Fractals 11,373 (2000).

[8] C. Beck, F. Schlögl, Thermodynamics of chaotic systems, Cambridge University Press, Cambridge (1993).

[9] Ya.B. Pesin, Russian Mathematical Surveys (4)32,55(1977).

[10] J.L. Lebowitz, Physica A 263, 516 (1999).

[11] D.J. Driebe, Fully Chaotic Maps and Broken Time Symmetry, Kluwer Academic Publishers, Dordrecht, The Netherlands, 1999.

[12] A. Lasota, M.C. Mackey, Chaos, Fractals and Noise, Stochastic Aspects and Dynamics, Second Edition, Springer-Verlag, New York (1994), p.13.

[13] M.C.Mackey, Time's Arrow:The Origin of Thermodynamic Behavior, Springer-Verlag, New York (1992).

[14] J.R. Dorfman, An Introduction to Chaos in Nonequilibrium Statistical Mechanics, Cambridge University Press, Cambridge (1999).

[15] C. Tsallis, A.R. Plastino, and W.-M.Zheng, Chaos, Soliton, Fractals,8, 885 (1997).

[16] L. Tessieri, D. Vitali, P. Grigolini, Phys. Rev. A 51, 4404 (1995). 
[17] E. Floriani, R. Mannella, P. Grigolini, Phys. Rev. E 52, 5910 (1995).

[18] M. Bologna, P. Grigolini, and J. Riccardi, Phys.Rev. E 82 , 6432 (1999).

[19] R.F. Fox, Chaos 8, 462 (1998).

[20] M. Abramowitz and I.A. Stegun, Handbook of Mathematical Functions, Dover, New York (1972) p. 804.

[21] A. Pattanayak and P. Brumer, Phys. Rev. E 56, 5174 (1997).

[22] A. Pattanayak and P. Brumer, Phys. Rev. Lett. 79, 4131 (1997).

[23] G. M. Zaslavsky, Physics of Chaos in Hamiltonian Systemson the Foundations of Statistical Physics,Imperial College Press, London (1988).

[24] G.M. Zaslavsky, Physics Today 52(8), 39 (1999).

[25] M. Ignaccolo, P. Grigolini, in preparation for Phys. Rev. E.

[26] C. Tsallis, J. Stat. Phys.52, 479 (1988). 


\title{
Trajectory versus probability density entropy
}

\author{
Mauro Bologna ${ }^{1}$, Paolo Grigolini ${ }^{1,2,3}$, Markos Karagiorgis ${ }^{1}$ \\ ${ }^{1}$ Center for Nonlinear Science, University of North Texas, \\ P.O. Box 5368, Denton, Texas 76203 \\ ${ }^{2}$ Istituto di Biofisica del Consiglio Nazionale delle \\ Ricerche, Via San Lorenzo 26, 56127 Pisa, Italy \\ ${ }^{3}$ Dipartimento di Fisica dell'Università di Pisa, \\ Piazza
}

Torricelli 2, 56127 Pisa, Italy

(April 10, 2000)

\begin{abstract}
We study the problem of entropy increase of the Bernoulli-shift map without recourse to the concept of trajectory and we discuss whether, and under which conditions if it does, the distribution density entropy coincides with the Kolmogorov-Sinai entropy, namely, with the trajectory entropy.

05.45.+b,03.65.Sq,05.20.-y
\end{abstract}

Typeset using REVTEX 


\section{INTRODUCTION}

The problem of establishing a connection between the Kolmogorov-Sinai (KS) entropy $[1,2]$ and the conventional entropy expressed in terms of probability density is an interesting problem that is attracting some attention in literature [3,4]. Early work on this subject goes back to the discussion of Goldstein and Penrose [5]: These authors, almost twenty years ago, established a connection between the KS entropy and a coarse-grained version of the distribution density entropy. The work of Ref. [5] is based on a formal and rigorous mathematical treatment which for this reason might have eluded the attention of physicists working on this subject $[3,4]$. Thus we restate the problem using intuitive arguments which also make it possible for us to account for the more recent literature on the subject. In fact, our heuristic treatment will allow us to relate the results of the more recent work of Latora and Baranger [4] to the earlier work of Zurek and Paz [3].

In addition to revisiting the problem of how to make the KS entropy emerge from a nonequilibrium dynamic picture [5], we shall touch also the intriguing problem of whether a thermodynamic perspective has to rest on the adoption of trajectories, as implied by the concept itself of KS entropy, or on the use of probability densities, advocated with strong arguments by Petrosky and Prigogine [6,7]. It is convenient to stress that the KS entropy $[1,2]$ is a property of a single trajectory. The phase space is divided into cells, each cell

being assigned a given label $\omega_{r}$. Then we define a sequence of symbols by means of a single trajectory: The sequence is determined assigning to any time step the label of the cell where the trajectory lies at that time step. The trajectory is supposed to be large enough as to yield reliable values for the probabilities determined through the numerical frequencies. This means that we fix a window of size $N$, and we move this window along the sequence. For any window position a string of symbols $\omega_{0}, \omega_{1}, \ldots \omega_{N-1}$ is determined. Moving the window of fixed size $N$ along the infinite sequence generated by the trajectory we have to evaluate how many times the same string of symbols appears, thereby leading us to determine the probability $p\left(\omega_{0}, \omega_{1}, \ldots \omega_{N-1}\right)$. The KS entropy is then defined by 


$$
h_{K S} \equiv \lim _{N \rightarrow \infty} H(N) / N
$$

where $H(N)$ is the conventional Shannon entropy of the window of size $N$ defined by

$$
H(N)=\sum_{\omega_{0}, \omega_{1} \ldots \omega_{N-1}} p\left(\omega_{0}, \omega_{1}, \ldots \omega_{N-1}\right) \ln \left[p\left(\omega_{0}, \omega_{1}, \ldots \omega_{N-1}\right)\right] .
$$

It is evident therefore that the KS entropy rests on trajectories, and, more specifically, it implies the adoption of only one trajectory of virtually infinite length. The KS entropy is very attractive because its value turns out to be independent of the repartition into cells of the phase space, due to the crucial role of the so called generating partitions [8]. In the specific case where a natural invariant distribution exists, it is shown [9] that

$$
h_{K S}=\sum_{i} \int d \mathbf{x} \rho_{e q}(\mathbf{x}) \lambda_{i}(\mathbf{x})
$$

with $\lambda_{i}(\mathbf{x})>0$. Note that $\mathbf{x}$ denotes the coordinate of a multidimensional phase space, $\rho_{e q}(\mathbf{x})$ is the natural invariant distribution and $\lambda_{i}(x)$ is a local Lyapounov coefficient, with $i=1, d, d$ being the dimension of the system under study. From Eq.(3) we see that, as earlier pointed out, the KS entropy is independent of the repartition into cells. The original definition of Eq.(1), with $N$ thought of as time, means that the KS entropy, as a property of a single trajectory, is the rate of entropy increase per unit of time. However, since the single trajectory under examination is infinitely long, and explores in time all the phase space available, the KS entropy can also be expressed in the form of an average over the equilibrium distribution density, without any prejudice for the single trajectory nature of this "thermodynamic" property.

According to Petrosky and Prigogine [6,7], on the contrary, the connection between dynamics and thermodynamics implies the use of the Liouville equation

$$
\frac{\partial}{\partial t} \rho(\mathbf{x}, t)=-i L \rho(\mathbf{x}, t)
$$

where $L$ denotes both the classical and the quantum Liouville operator, and $\rho(\mathbf{x}, t)$ is the nonequilibrium distribution density. The reason for this choice is that the analysis of the Liouville operator, through the "rigged Hilbert" space, allows the appearance of complex 
eigenvalues which correspond to irreversibility, and to the collapse of trajectories as well. This is the reason why distribution densities are judged to be more fundamental than trajectories.

In this paper we limit our analysis to the special case where dynamics are generated by maps rather than by Hamiltonians. We do not address the difficult issue of discussing the thermodynamic limit $N \rightarrow \infty$ which is the subject of very interesting recent discussions $[7,10]$, and where, according to Lebowitz [10], ergodicity and mixing are neither necessary nor sufficient to guarantee the connection between dynamics and thermodynamics. We consider the case of low-dimension chaos, where probability emerges as a consequence of sensitivity to initial conditions [7]. Even in this case, however, according to the perspective established by Petrosky and Prigogine [6,7], probability densities are more fundamental than trajectories. The readers interested in knowing more about this perspective, entirely based on probability density, should consult the illuminating work of Driebe [11]. In this case the counterpart of Eq.(4) becomes

$$
\rho(\mathbf{x}, t+1)=\Lambda \rho(\mathbf{x}, t)
$$

where $\Lambda$ is referred to as Frobenius-Perron operator. Of course, the operator $L$ of Eq.(4) has to be identified with $i(\Lambda-1)$.

According to the traditional wisdom, the Frobenius-Perron operator is expected to make the distribution densities evolve in the same way as that resulting from the time evolution of a set of trajectories with initial conditions determined by the initial distribution density [11]:The known cases of discrepancy between the two pictures are judged to be more apparent than real [12]. Nevertheless, even in the case of invertible maps, the birth of irreversibility can be studied using the same perspective as that adopted for Hamiltonian systems, with Eq.(4) replaced by Eq.(5), and so using again probability densities rather than trajectories.

However, we attempt at digging out the KS entropy from Eq.(5), and this purpose forces us to formulate a conjecture on how to relate entropy to $\rho(\mathbf{x}, t)$. A plausible choice seems to be 


$$
S(t)=-\int_{\mathbf{X}} \rho(\mathbf{x}, t) \ln [\rho(\mathbf{x}, t)] d \mathbf{x}
$$

We share the view of Goldstein and Penrose [5] who consider the KS entropy to be a nonequilibrium entropy. In other words, we may hope to derive the KS entropy from the time derivative of $S(t)$ of Eq.(6). As Goldstein and Penrose do [5], to realize that purpose we have to address a delicate problem: In the case of invertible maps, $S(t)$ is time independent [13], thereby implying a vanishing KS entropy. Yet, the baker's transformation, which is a well known example of invertible map, thereby yielding a time independent $S(t)$, is shown [14] to yield a KS entropy equal to $\ln 2$, a fact suggesting a steady condition of entropy increase. We plan to discuss all this with the joint use of heuristic arguments and of the rigorous theoretical tools of Ref. [11].

The present paper uses as a paradygm of invertible map the two-dimensional baker's transformation, depending on two coordinates, $x$ and $y$, the former corresponding to dilatation and the latter to contraction. Using this prototype for invertible dynamics, we aim at proving that the adoption of the distribution density in the case of invertible chaotic maps would lead to an increasing process of fragmentation, depending not only, as the KS entropy does, on the positive Lyapounov coefficient, but also on the negative one. The adoption of a coarse graining has the effect of quenching the action of the negative Lyapunov coefficient, thereby allowing the KS entropy to show up. Then, to go beyond these heuristic arguments we make a trace on the variable $y$, namely, on the process responsible for contraction, and we focus our attention on the contracted dynamics. This is equivalent to that produced by the Bernoulli shift map. Here room is only left for dilatation and the problem can be solved with a rigorous mathematical method, without using trajectories.

The outline of the paper is as follows. In Section II we shall illustrate our heuristic picture. In Section III we shall address the problem by means of a rigorous treatment resting on the theoretical tools provided by Driebe [11]. In Section IV we shall draw some conclusions. Some delicate mathematical problems behind the theoretical calculations of Section III are detailed in Appendix. 


\section{HEURISTIC ARGUMENTS}

Note that the cases studied by Latora and Baranger [4] are two-dimensional, and our discussion here refers to a two-dimensional case, too. We have in mind the backer's transformation and $\mathbf{x} \equiv(x, y)$. We denote by $W(t)$ the number of cells occupied at a given time $t$. Note that $W(0)<W_{\max }$, where the symbol $W_{\max }$ denotes the total number of cells into which we have divided the phase space $\mathbf{X}$. Our heuristic approach is based on the following assumptions.

(i) At the initial time only $W(0)$ cells are occupied.

(ii) At all times the trajectories are equally distributed over the set of occupied cells. This means

$$
S(t)=\ln W(t) .
$$

(iii) We denote by $\lambda$ the positive Lyapounov coefficient, and we set

$$
W(t)=W(0) \exp (\lambda t) .
$$

All these three assumptions have been borrowed from the recent work of Ref. [15]. The joint use of all them yields

$$
S(t)=\lambda t-\ln W(0),
$$

which corresponds to the Kolmogorov thermodynamical regime. Note that the positive Lyapounov coefficient in the case of the baker's transformation is shown [14] to be:

$$
\lambda=\ln 2 .
$$

Note also that according to the arguments of Section I, the connection with the KS entropy is established through the time derivative of $S(t)$. Thus, we conclude that

$$
\frac{d S}{d t}=\lambda=\ln 2=h_{K S},
$$

which corresponds to deriving the KS entropy from the distribution density picture. 
This Kolmogorov regime is not infinitely extended. It has an upper bound, given by the fact that when equilibrium is reached, even in the merely sense of a coarse-grained equilibrium, then the entropy stops increasing. An estimate of this time is obviously given by the solution of the following equation

$$
\ln W_{\max }=\lambda t-\ln W(0)
$$

which yields the following saturation time

$$
t_{S}=\frac{1}{\lambda} \ln \left(\frac{W_{\max }}{W(0)}\right)
$$

Furthermore a lower bound of validity exists, which will be easily estimated with very simple arguments. If the initial distribution includes a large number of cells and the size of this distribution along the coordinate $y$ is $L$, and the size of the cells is $\epsilon$ with $\epsilon<L$, then it is evident that, in spite of the coarse graining the total number of cells occupied remains the same for a while. This time is easily estimated using the equation

$$
\operatorname{Lexp}(-\lambda t)=\epsilon
$$

which in fact defines the time at which the distribution volume, and consequently, the system entropy starts increasing. This time is denoted by the symbol $t_{D}$ and reads

$$
t_{D}=\frac{1}{\lambda} \ln \left(\frac{L}{\epsilon}\right)
$$

We denote by $U(t)$ the volume of the distribution density at time $t$ and by $V$ the volume of the phase space, thereby implying that $U(t) \leq V$. We note that

$$
\frac{W_{\max }}{W(0)}=\frac{V}{U(0)}
$$

where $V$ is the total volume of the phase space and $U(0)$ is the initial volume of the distribution density. Thus the Kolmogorov regime shows up in the following time interval

$$
t_{D}=\frac{1}{\lambda} \ln \left(\frac{L}{\epsilon}\right)<t<t_{S}=\frac{1}{\lambda} \ln \left(\frac{V}{U(0)}\right) .
$$


The time duration of the regime of validity of the Kolmogorov regime can be made infinitely extended by making the cell size infinitely small. This means that the conflict between the KS entropy prescription and the time independence of $S(t)$ can be bypassed by focusing our attention on the intermediate region, whose time duration tends to infinity with $\epsilon \rightarrow 0$. We note that a choice can be made such that $V / U(0)=(L / \epsilon)^{\chi}$, with $\chi>1$. This means the time duration of the Kolmogorov regime can be made $\chi$ times larger than the time duration of the transition regime. For $\epsilon \rightarrow 0$ both time durations become infinite, thereby showing that a Kolmogorov regime of infinite time duration can be obtained at the price, however, of waiting an infinitely long time for the entropy to increase. The infinite waiting time before the regime of entropy increase fits the observation [13,12] that the Gibbs entropy of an invertible map is constant. The linear entropy increase showing up "after this infinite waiting time" allows the emergence of the KS entropy from within the probability density perspective.

This kind of coarse graining might be criticized as corresponding to arbitrary choices of the observer. It is interesting to remark that there exists another interesting form of coarse graining, produced by weak stochastic forces. Both in the case where this stochastic forces mimic the interaction with the environment [3] or in the case where it happens to be an expression of spontaneous fluctuations [16] this kind of coarse graining can be regarded as being produced by nature. Here we limit ourselves to remarking that according to Zurek and Paz [3] these stochastic forces contribute a fluctuation-dissipation process mimicking the interaction between the system of interest and the environment. These authors studied the inverted stochastic oscillator

$$
\frac{d^{2} x}{d t^{2}}=\lambda^{2} x(t)+\gamma \frac{d x}{d t}+f(t)
$$

where the friction $\gamma$ and the stochastic force $f(t)$ are related to one another by the standard fluctuation-dissipation relation

$$
<f f(t)>=2 \gamma<\left(\frac{d x}{d t}\right)^{2}>_{e q} \delta(t) \equiv 2 D \delta(t)
$$


It is interesting to remark that the proper formulation of the second principle implies that the entropy of a system can only increase or remain constant under the condition of no energy exchange between the system and its environment. In the case of Eq.(19) the energy exchange between system and environment is negligible for any observation made in the time scale

$$
t<<1 / \gamma
$$

To ensure that the system entropy increase to take place with no energy exchange between system and its environment Zurek and Paz [3] set the condition of Eq.(20) and this, in turn, allows them to neglect the friction term in Eq.(18). Then, these authors adopted the modes

$$
u \equiv \frac{d x}{d t}+\lambda x
$$

and

$$
w \equiv \frac{d x}{d t}+\lambda x
$$

which make it possible for them to split Eq.(18) into

$$
\frac{d u}{d t}=\lambda u(t)+f(t)
$$

and

$$
\frac{d w}{d t}=-\lambda w(t)+f(t)
$$

Let us imagine the initial distribution density as a rectangle of size $\Delta w(0)$ along the direction $w$ and $\Delta u(0)$ along the direction $u$. We keep denoting by $U(t)$ the distribution volume at a given time $t$. Thus the volume of the initial distribution is

$$
U(0)=\Delta u(0) \Delta w(0)
$$

In the absence of the stochastic force $f(t)$, Eqs.(23) and Eqs.(23) result in an exponential increase and an exponential decrease, with the same rate $\lambda$, respectively. Consequently, the 
Liouville theorem $U(t)=U(0)$ is fulfiled. In the presence of stochastic force, we work as follows. In the former equation, with $u$ increasing beyond any limit, the weak stochastic force $f(t)$ can be neglected. This is not the case with the latter equation. In fact, $w$ is a contracting variable in the absence of the stochastic force. In the presence of the stochastic force the minimum size of the distribution along $w$ is given by

$$
<w^{2}>_{e q}^{1 / 2}=(D / \lambda)^{1 / 2}
$$

This minimum size is reached in a time determined by the solution of the following equation

$$
\Delta w(0) \exp (-\lambda t)=(D / \lambda)^{1 / 2}
$$

yielding

$$
t_{D}=\frac{1}{\lambda} \ln \left(\frac{\lambda}{D}\right)^{1 / 2} \Delta w(0)
$$

Due to the fact that deterministic chaos is simulated by Zurek and Paz [3] by means of an inverted parabola, these authors did not consider the entropy saturation effects. However, it is straigthforward to evaluate the saturation effect with heuristic arguments concerning the case where the total volume of the phase space has the finite value $V$. From the time $t=t_{D}$ on, the distribution volume $U(t)$ increases exponentially in time with the following expression

$$
U(t)=\Delta w(0) \Delta u(0) \exp (\lambda t)=(D / \lambda)^{1 / 2} \Delta u\left(t_{D}\right) \exp (\lambda t)
$$

Thus, the saturation time is now given by

$$
t_{S}=\frac{1}{\lambda} \ln \left[\frac{V}{\Delta u(0) \Delta w(0)}\right] .
$$

Using Eq.(25) we can write this saturation time as

$$
t_{S}=\frac{1}{\lambda} \ln \left[\frac{V}{U(0)}\right]
$$

which coincides with Eqs.(17) and (13). 
In conclusion, it seems that the emergence of a Kolmogorov regime is made possible by the existence of a form of coarse graining, and that it is independent of whether the coarse graining is realized by the division into cells or by a weak stochastic force. This property seems to make less important the discussion of whether the stochastic force is of environmental origin or rests on some kind of extension of the current physical laws. However, we have to point out that the situation significantly changes, if we move from a strongly to a weakly chaotic classical system. As a relevant example, let us refer ourselves to the work of Ref. [17]. The authors of this work study the asymptotic time limit of a diffusion process generated by using an intermittent map as a dynamic generator of diffusion. If these dynamics are perturbed by a white noise, a transition is provoked, at long times, from anomalous to normal diffusion. When the only source of random behavior is given by the sporadic randomness of the intermittent map [18], the long-time limit is characterized by Lévy statistics, a physical condition in a striking conflict with the condition of Gaussian statistics produced by the action of fluctuations [17]. Here we limit our attention to the case of strong chaos where the two distinct sources of coarse graining produce equivalent effects. It might be of some interest for the reader to compare the coarse-graining approach of this section to the more formal method recently adopted by Fox [19] to deal with the same problem.

It is interesting to stress that to make the regime of validity of the Kolmogorov regime as extended as possible we must make the ratio $V / U(0)$ as large as possible (virtually infinite). This means that we have to choose an initial distribution density so sharp as to become apparently equivalent to a single trajectory. This seems to be an attractive way of explaining why in this condition the KS entropy is recovered, since, as stressed in Section I, the KS entropy is a single trajectory property. However, in accordance with the authors of Refs. $[6,7,11]$ we must admit that there exists a deep difference between a trajectory and a very sharp distribution. The latter is stable and robust, while the former is not. In Section III we shall show that the rigorous derivation of the Kolmogorov regime requires a non trivial mathematical procedure, and the mathematical effort to make from this side, to derive the 
KS entropy, serves the useful purpose of proving that the KS entropy of a trajectory is a really wise way of converting into advantages the drawbacks of the trajectory instability.

\section{THE KS ENTROPY FROM A REDUCED FROBENIUS-PERRON EQUATION}

This Section is devoted to a rigorous discussion resting only on the theoretical tools described in Ref. [11] for a genuine probability density aproach. According to Mackey [13], if we rule out the possibility that the laws of physics are misrepresented by invertible dynamic

prescriptions, there are only two possible sources of entropy increase. The first is the coarse graining discussed in Section II. The second is the adoption of reduced equation of motion, obtained by a trace over "irrelevant" degrees of freedom [13]. In fact here we study the Bernoulli shift map,

$$
x_{t+1}=2 x_{t}, \bmod 1 \text {. }
$$

The Frobenius-Perron equation of this map is defined by [11]

$$
\rho(x, t+1)=\Lambda \rho(x, t) \equiv \frac{1}{2}\left[\rho\left(\frac{x}{2}, t\right)+\rho\left(\frac{x+1}{2}, t\right)\right]
$$

It is straigtforward to show that the Frobenius-Perron operator of Eq.(33) stems from the contraction over the variable $y$ of the baker's mapping, acting in fact on the unit square of two-dimensional space (x,y) (see, for instance Ref. [14]). It is shown [14] that the KS entropy of the baker's transformation is well defined and turns out to be the same as that of the Bernoulli shift map, namely $h_{K S}=\ln 2$. Intuitively, this suggests that the main role of coarse graining is that of making inactive the process of contraction, and with it the negative Lyapunov coeficient. This intuitive argument seems to be plausible and raises the interesting question of how to prove it with a rigorous approach. This is equivalent to deriving the Kolmogorov regime using a rigorous mathematical method rather than the heuristic arguments of Section II. We must observe again that this is made possible by the fact that the tracing has changed the originally invertible map into one that is not invertible. 
To address this issue we follow the prescription of Ref. [11]. First of all, we express the distribution density at time $t$ under the form given by Ref. [11] which reads:

$$
\rho(x, t)=1+\sum_{j=1}^{\infty} \exp \left(-\gamma_{j} t\right) \frac{B_{j}(x)}{j !}\left[\rho^{(j-1)}(1,0)-\rho^{(j-1)}(0,0)\right] .
$$

Note that $\gamma_{j} \equiv j \ln 2, B_{j}(x)$ are the Bernoulli polynomials [20] and $\rho^{(n)}(x, t)$ denotes the $n$-th order derivative of $\rho(x, t)$ with respect to $x$. Hereby, we shall show how to derive from the previous one more tractable expression, which will be checked in appendix.

In the case of an initial condition close to equilibrium, resulting from the sum of the equilibrium distribution and the first "excited" state, it is easy to prove that the entropy $S(t)$ of Eq. (6) reaches exponentially in time the steady-state condition. This suggests that the Kolmogorov regime, where the entropy $S(t)$ is expected to be a linear function of time, must imply an initial condition with infinitely many "excited" states. To deal with a condition of this kind it is convenient to express Eq.(34) in an equivalent form given by

$$
\rho(x, t)=1+\sum_{j=1}^{\infty} \int_{-\infty}^{+\infty} \frac{B_{j}(x)}{j !}(-i z \omega)^{j-1} \hat{\rho}(\omega)(\exp (-i \omega)-1) \frac{d \omega}{2 \pi},
$$

where $z \equiv \exp [-t(\ln 2)]$ and $\hat{\rho}(\omega)$ is related to the initial condition $\rho(x, 0)$ by the Fourier transform

$$
\rho(x, 0)=\int_{-\infty}^{+\infty} \hat{\rho}(\omega) \exp (-i \omega x) \frac{d \omega}{2 \pi}
$$

The following equation

$$
\sum_{j=0}^{\infty} \frac{B_{j}(x)}{j !} z^{j}=z \frac{\exp (z x)}{\exp (z)-1}
$$

is known [20] to generate Bernoulli polynomials. Using this Bernoulli polynomial generatrix, we arrive, after some algebra, at

$$
\rho(x, t)=z \int_{-\infty}^{+\infty} \exp (-i \omega z x) \hat{\rho}(\omega) \frac{\exp (-i \omega)-1}{\exp (-i \omega z)-1} \frac{d \omega}{2 \pi}
$$

By expanding the denominator of Eq.(38) into a Taylor series and using Eq.(36), we finally derive the fundamental expression 


$$
\rho(x, t)=z \sum_{n=0}^{\infty}[\rho(z x+z n, 0)-\rho(z x+z n+1,0)] .
$$

This important expression makes it possible for us to discuss analytically the entropy time evolution ensuing the preparation of an initially very sharp distribution. Let us consider in fact

$$
\rho(x, 0)=\frac{\alpha}{1-\exp (-\alpha)} \exp (-\alpha x), 0 \leq x \leq 1 .
$$

For $\alpha \rightarrow \infty$ this initial distribution becomes a very sharp distribution located at $x=0$. By plugging this initial distribution density into Eq.(39) we obtain

$$
\rho(x, t)=z \alpha \frac{\exp (-\alpha x z)}{1-\exp (-\alpha z)}
$$

It is evident that this simple analytical expression for the time evolution of the distribution density is exact, and corresponds to the time evolution dictated by the Frobenius-Perron operator of Eq.(33).

We are now in a position to discuss the central issue of this paper, namely, the time evolution of the Gibbs entropy of Eq.(6), which, in the case here under study, reads

$$
S(t)=-\int_{X} \rho(x, t) \ln [\rho(x, t)] d x
$$

with $X$ now denoting the interval [0,1]. By plugging Eq.(40) within Eq.(42) we obtain

$$
S(t)=1-\ln (\alpha z)+\ln [1-\exp (-\alpha z)]-\frac{\alpha z}{\exp (\alpha z)-1}
$$

In the limiting case $\alpha \rightarrow \infty$ this exact prediction is approximated very well by

$$
S(t)=-\ln (\alpha)+(\ln 2) t
$$

It indicates that a sharp initial distribution makes the system evolve according to the KS entropy, with no regime of transition from mechanics to thermodynamics. The third regime of Ref. [4] is still present. It is straigtforward to show that the saturation time $t_{S}=\ln \alpha / \ln 2$ resulting from Eq.(43) is the same as that of Eq.(13) in the case $V=1$. In fact using 
Eq.(16) and $V=1$ we obtain that $W_{\max } / W(0)=1 / U(0)$, where $U(0)$ is the size of the initial distribution. The size of the initial distribution of Eq.(40), for $\alpha \rightarrow \infty$, becomes prportional to $1 / \alpha$. Thus, $\ln \alpha \approx \ln \left(W_{\max } / W(0)\right.$ in accordance with Eq. (13).

This is an elegant result, involving a modest amount of algebra. However, it refers to an initial distribution located at $x=0$. We want to prove that this is a general property, independent of where the initially sharp distribution is located, at the price, as we shall see, of a more complicated mathematical treatment. For this purpose we study the case where the distribution shape is the Lorentzian curve:

$$
\rho(x, 0)=A \frac{\Gamma}{\left(x-x_{0}\right)^{2}+\Gamma^{2}},
$$

with $x_{0}$ being a generic point of the interval $[0,1]$ and $x$ running in the same interval. Setting the normalization condition yields

$$
A=\frac{1}{\arctan \left(\frac{x_{0}}{\Gamma}\right)+\arctan \left(\frac{1-x_{0}}{\Gamma}\right)} .
$$

We have to set again the condition that the initial distribution is very sharp. Thus we make the assumption $\Gamma \rightarrow 0$, yielding $A \approx 1 / \pi$. We plug this approximated value of $A$ into Eq. (39), thereby obtaining the following density time evolution

$$
\rho(x, t)=\frac{z \Gamma}{\pi} \sum_{n=0}^{\infty}\left[\frac{1}{\left(z x+z n-x_{0}\right)^{2}+\Gamma^{2}}-\frac{1}{\left(z x+z n-x_{0}+1\right)^{2}+\Gamma^{2}}\right] .
$$

We are now in a position to study the entropy time evolution again. Plugging Eq.(47) into (42) we find

$$
\begin{array}{r}
S(t)=-\int_{X} \rho(x, t) \ln \left[\frac{z}{\pi \Gamma} \sum_{n=0}^{\infty} \frac{1}{\left(\frac{z x+z n-x_{0}}{\Gamma}\right)^{2}+1}-\frac{1}{\left(\frac{z x+z n-x_{0}+1}{\Gamma}\right)^{2}+1}\right] d x \\
=-\int_{X} \rho(x, t) \ln \left[\frac{z}{\pi \Gamma}\right] d x-\int_{X} \rho(x, t) \ln \left[\sum_{n=0}^{\infty} \frac{1}{\left(\frac{z x+z n-x_{0}}{\Gamma}\right)^{2}+1}-\frac{1}{\left(\frac{z x+z n-x_{0}+1}{\Gamma}\right)^{2}+1}\right] d x,
\end{array}
$$

where $[y]$ denotes the integer part of $y$. To derive a more tractable expression we note that in the limiting case of $\Gamma$ very small, the quantities [...] contributing this series are almost 
zero except for $n=-[x]+\left[\frac{x_{0}}{z}\right]=\left[\frac{x_{0}}{z}\right]$ in the first term, and for the possible contribution

$n=-[x]-\left[\frac{1-x_{0}}{z}\right]=-\left[\frac{1-x_{0}}{z}\right]$ in the second term. The latter condition cannot be realized, since $n$ is a positive integer. Thus, using only the first class of contributions, we get for the entropy $S(t)$ the following approximate expression

$$
S(t) \approx \ln \Gamma+(\ln 2) t-\ln \pi-\int_{0}^{z / \Gamma} \frac{\ln \left(y^{2}+1\right)}{y^{2}+1} d y
$$

which, in the limiting case $\frac{z}{\Gamma} \rightarrow \infty$ becomes

$S(t) \approx \ln \Gamma+(\ln 2) t-\ln \pi-\int_{0}^{\infty} \frac{\ln \left(y^{2}+1\right)}{y^{2}+1} d y=\ln \Gamma+(\ln 2) t-\ln \pi-\pi \ln 2 \approx \ln \Gamma+(\ln 2) t$.

As in the earlier case, the validity of the approximation yielding the linear dependence of $S(t)$ on time, is broken at the time $t \sim-\ln \Gamma / \ln 2$. In conclusion, the Kolmogorov condition is realized by very sharp initial distributions. Our derivation of this interesting property was done adhering to the recommendation of Ref. $[6,7,11]$ of resting only on densities rather than on trajectories.

\section{CONCLUDING REMARKS}

This paper shows that there exists a subtle difference between a Liouville density and a probability distribution. The Liouville distribution density of a chaotic map, which is at the same time invertible, becomes increasingly fragmented with time. If the initial distribution has a volume which is much smaller than the volume of the phase space, the highly fragmented distribution density at large times has a volume identical to the initial, but the impression afforded by a coarse-grained observation is that the initial volume increases with time till to become equal to that of the whole phase space. If we do the calculation of the time evolution of the Liouville density observing the motion of many trajectories with slightly different initial conditions, we are forced to adopt a coarse-graining procedure, dictated by the need itself of counting how many trajectories are found at a given time in 
a given small region of the phase space. This has the effect of making the Gibbs entropy increase. If, on the contrary, the calculation genuinely rests on the motion of the Liouville density, thereby implying that a quantum-like formalism is adopted, the Gibbs entropy is constant.

On the other hand, the KS entropy is a trajectory property and this, to first sight, might lead us to believe that, being a trajectory property, cannot be recovered from within an approach genuinely resting on the distribution density. The heuristic arguments used in Section II subtly rest on the trajectory properties and thus on the assumption that the two perspectives are equivalent in spite of the warnings of the authors of Refs. $[6,7,11]$. This is the reason why we judge the theoretical calculations of Section III to be of significant interest. These results have been obtained without having any direct recourse to the trajectory instability and only using the theoretical tools illustrated in Ref. [11], which in fact address the problem of the dynamics of map using the quantum mechanical language of eigenstate and eigenvalue. It has to be pointed out that to establish this rigorous connection between the linear increase of the Gibbs entropy and the KS entropy we need to use infinitely many "excited" states, namely, a condition very far from equilibrium. The adoption of a "reduced" Liouville-like equation has been essential for the success of this enterprise. The Frobenius-Perron operator of the Bernoulli shift map is obtained from the Frobenius-Perron operator of the baker's transformation via contraction over the variable $y$, corresponding to the contraction process. As a consequence, room is only left for dilatation. This is the reason why the heuristic argument of Eq.(8) holds true with no restriction and the use of a method rigorously based on densities lead to the same result. After all, the adoption of the theoretical tools of Ref. [11] as a rigorous way to evaluate the regression to equilibrium, must reflect in a way the trajectory instabilities behind the Pesin theorem of Eq.(3).

In the case where the action of the negative Lyapounov coefficient is quenched by a coarse-graining process, in addition to the saturation and to the Kolmogorov regime, also a short-time regime of transition to thermodynamics appears, so that, as found in Ref. [4] three distinct regimes can be detected, the regime of transition to thermodynamics, 
the Kolmogorov regime, and the saturation regime. Here we show that the three regimes discussed by Latora and Baranger [4] are exhibited also in the case of a coarse graining produced by random fluctuations.

In conclusion, this paper contributes to deepening our understanding of the connection between density and trajectory distribution. The density entropy exhibits a regime of increase linear in time with a rate equivalent to that of the trajectory entropy if the initial distribution is very sharp. It has to stressed that it cannot be infinitely sharp. This would make the distribution density useless, since an infinitely sharp initial distribution would manifest the lack of robustness pointed out by Driebe [11]. Thus, the connection is established using a genuine density. At the same time the accordance between the heuristic approach yielding the derivation of Eq.(44) can be interpreted as a rigorous support of the assumptions made by some some authors [15] to derive the KS entropy from within a probability distribution approach.

We want to remark that we are not aware of earlier work where the Lyapounov coefficient is derived analytically from a probability density approach with no use of heuristic arguments but that of Pattanayak and Brumer [21] which, however, seems to be limited to the study of the transition regime [22].

A problem open to future research concerns the role of coarse-graining. We have seen that in the case of strong chaos studied in this paper there is no essential difference between the coarse graining resulting from the repartition of the phase space into cells and the coarse graining caused by fluctuations. In the case of dynamic systems like the weakly chaotic billiards studied by Zaslavsky [23,24], which are easily proved to be statistically equivalent to the intermittent maps of Ref. [17], the two different coarse-graining sources result in different physical effects at long times. On the other hand, it is expected [25] that the dynamic process of transition to Lévy statistics is characterized by thermodynamic properties of non-extensive nature [26]. The study of the time evolution of non-extensive entropy under the actions of the two different sources of coarse graining studied in this paper would an interesting program for future research work. 


\section{APPENDIX}

The purpose of this Appendix is to check the main result of Section III. Our theoretical reference on this issues is given by the book of Ref. [11]. We note, however, that Eqs. (41) and Eq.(47) are not directly derived from Eq.(34), which is a theoretical finding of Ref. [11], but they are derived from Eq.(39), wich is the result of a further development of the theory of Ref. [11]. We feel therefore the need of proving that Eq.(39) fits the main requirement of keeping the norm unchanged and of being an exact solution of Frobenius-Perron equation of Eq.(33). To double check our results, we shall prove also that Eqs. (41) and Eq.(47) fit the same property. As a general remark about the content of this appendix we note that a function of the variable $x$, defined only within the finite interval $[0,1]$, admits a treatment based on its Fourier transform if it is thought of as being defined on the whole interval $[-\infty, \infty]$ with vanishing value outside $[0,1]$. Similarly we can define the Fourier series of this function assuming it to be periodically repeated all over the real axis. We shall adopt this approach throughout the whole appendix.

Let us check Eq.(39) first. We note that the argument of the density of Eq.(39) can be arbitrary with the only condition that the variable $x$ is in the interval $[0,1]$. Furthermore, since Eq.(39) is derived from Eq.(38), it is enough for us to prove that Eq.(38) is properly normalized and is a solution of the Frobenius-Perron equation of Eq.(33). In conclusion, we have to check:

$$
\rho(x, t)=z \int_{-\infty}^{+\infty} e^{-i \omega z x} \hat{\rho}(\omega) \frac{e^{-i \omega}-1}{e^{-i \omega z}-1} \frac{d \omega}{2 \pi} .
$$

First we check that this equation is norm conserving or:

$$
\int_{0}^{1} \rho(x, t) d x=1
$$

To do so, we integrate Eq. (A-1 with respect to the variable $\mathrm{x}$ from $-\infty$ to $+\infty$. Thus we obtain: 


$$
\begin{aligned}
& \int_{0}^{1} z \int_{-\infty}^{+\infty} e^{-i \omega z x} \hat{\rho}(\omega) \frac{e^{-i \omega}-1}{e^{-i \omega z}-1} \frac{d \omega}{2 \pi} d x \\
= & \int_{-\infty}^{+\infty} \frac{e^{-i \omega z}-1}{-\imath \omega} \hat{\rho}(\omega) \frac{e^{-i \omega}-1}{e^{-i \omega z}-1} \frac{d \omega}{2 \pi} .
\end{aligned}
$$

This means the expression

$$
\int_{-\infty}^{+\infty} \frac{e^{-i \omega}-1}{-\imath \omega} \hat{\rho}(\omega) \frac{d \omega}{2 \pi} \equiv \int_{0}^{1} \int_{-\infty}^{+\infty} e^{-i \omega y} \hat{\rho}(\omega) \frac{d \omega}{2 \pi} d y
$$

The integral over $\omega$ is by definition the Fourier transform of $\rho(y, 0)$, yielding thereby

$$
\int_{0}^{1} \rho(x, t) d x=\int_{0}^{1} \rho(y, 0) d y=1 \forall t
$$

due to the fact that the initial condition is assumed to be normalized.

We want now to prove that the distribution density $\rho(x, t)$ of Eq. (A-1) is a solution of the Frobenius-Peron operator of Eq.(33), namely, that:

$$
\rho(x, t+1)=\frac{1}{2}\left[\rho\left(\frac{x}{2}, t\right)+\rho\left(\frac{x+1}{2}, t\right)\right] .
$$

Remembering that $z=2^{-t}$ and $\frac{z}{2}=2^{-t-1}$ we can write Eq.(A-1)as:

$$
\rho(x, t+1)=\frac{z}{2} \int_{-\infty}^{+\infty} e^{-i \omega z x / 2} \hat{\rho}(\omega) \frac{e^{-i \omega}-1}{e^{-i \omega z / 2}-1} \frac{d \omega}{2 \pi}
$$

Plugging Eq.(A-1) into it, the r.h.s. of Eq.(A-6) becomes:

$$
\begin{gathered}
\frac{1}{2}\left[\rho\left(\frac{x}{2}, t\right)+\rho\left(\frac{x+1}{2}, t\right)\right] \\
=\frac{z}{2}\left[\int_{-\infty}^{+\infty} e^{-i \omega z x / 2} \hat{\rho}(\omega) \frac{e^{-i \omega}-1}{e^{-i \omega z}-1} \frac{d \omega}{2 \pi}+\int_{-\infty}^{+\infty} e^{-i \omega z(x+1) / 2} \hat{\rho}(\omega) \frac{e^{-i \omega}-1}{e^{-i \omega z}-1} \frac{d \omega}{2 \pi}\right]
\end{gathered}
$$

and after a little algebra we get:

$$
\frac{1}{2}\left[\rho\left(\frac{x}{2}, t\right)+\rho\left(\frac{x+1}{2}, t\right)\right]=\frac{z}{2} \int_{-\infty}^{+\infty} e^{-i \omega z x / 2} \hat{\rho}(\omega) \frac{\left[e^{-i \omega}-1\right]\left[e^{-i \omega z x / 2}+1\right]}{e^{-i \omega z}-1} \frac{d \omega}{2 \pi} .
$$

By decomposing the denominator as follows 


$$
\frac{1}{e^{-i \omega z}-1}=\frac{1}{\left[e^{-i \omega z / 2}-1\right]\left[e^{-i \omega z / 2}+1\right]}
$$

and simplifying, we obtain

$$
\frac{1}{2}\left[\rho\left(\frac{x}{2}, t\right)+\rho\left(\frac{x+1}{2}, t\right)\right]=\frac{z}{2} \int_{-\infty}^{+\infty} e^{-i \omega z x / 2} \hat{\rho}(\omega) \frac{e^{-i \omega}-1}{e^{-i \omega z / 2}-1} \frac{d \omega}{2 \pi}
$$

that coincide with Eq.(A-7).

To check the property $\rho(x, t) \rightarrow 1$ for $t \rightarrow \infty$ or $z \rightarrow 0$ we use (A-1) and we write:

$$
\begin{gathered}
\rho(x, t) \sim z \int_{-\infty}^{+\infty} \hat{\rho}(\omega) \frac{e^{-i \omega}-1}{-i \omega z} \frac{d \omega}{2 \pi} \\
=\int_{-\infty}^{+\infty} \hat{\rho}(\omega) \frac{e^{-i \omega}-1}{-i \omega} \frac{d \omega}{2 \pi}=\int_{-\infty}^{+\infty} \hat{\rho}(\omega) \int_{0}^{1} e^{-i \omega x} d x \frac{d \omega}{2 \pi}=\int_{0}^{1} \rho(x) d x=1 .
\end{gathered}
$$

Now we shall test directly the Eq.(41) using Eq.(34). Plugging directly the initial distribution:

$$
\rho(x, 0)=\frac{\alpha}{1-e^{-\alpha}} e^{-\alpha x}, 0 \leq x \leq 1
$$

into Eq.(34) we obtain:

$$
\begin{aligned}
& \rho(x, t)=\sum_{j=0}^{\infty} e^{-\gamma_{j} t} \frac{B_{j}(x)}{j !}\left[\rho^{(j-1)}(1,0)-\rho^{(j-1)}(0,0)\right] \\
= & \frac{\alpha}{1-e^{-\alpha}} \sum_{j=0}^{\infty} e^{-\gamma_{j} t} \frac{B_{j}(x)}{j !}\left[\left(-\alpha^{j-1}\right) e^{-\alpha}-\left(-\alpha^{j-1}\right)\right] .
\end{aligned}
$$

Using the Bernoulli polynomials generatrix [20] we get

$$
\rho(x, t)=z \alpha \frac{e^{-\alpha x z}}{1-e^{-\alpha z}} .
$$

that coincides with Eq.(41) obtaned using the formula Eq.(39)

Finally let us to check the norm conservation of Eq.(47). Without any approximation, using the value of $A$ of Eq.(46), and a little algebra, we get:

$$
\begin{gathered}
\int_{0}^{1} \rho(x, t) d x=\left[\arctan \left(\frac{x_{0}}{\Gamma}\right)+\arctan \left(\frac{1-x_{0}}{\Gamma}\right)\right]^{-1} \\
\cdot \int_{0}^{1} z \Gamma \sum_{n=0}^{\infty}\left[\frac{1}{\left(z x+z n-x_{0}\right)^{2}+\Gamma^{2}}-\frac{1}{\left(z x+z n-x_{0}+1\right)^{2}+\Gamma^{2}}\right] d x \\
=\left[\arctan \left(\frac{x_{0}}{\Gamma}\right)+\arctan \left(\frac{1-x_{0}}{\Gamma}\right)\right]^{-1} \cdot \sum_{n=0}^{+\infty}\left[\arctan \left(\frac{x z+n z-x_{0}}{\Gamma}\right)-\arctan \left(\frac{x z+n z+1-x_{0}}{\Gamma}\right)\right]_{x=0}^{x=1} .
\end{gathered}
$$


This yields:

$$
\begin{gathered}
\int_{0}^{1} \rho(x, t) d x=\left[\arctan \left(\frac{x_{0}}{\Gamma}\right)+\arctan \left(\frac{1-x_{0}}{\Gamma}\right)\right]^{-1} \\
\cdot \sum_{n=0}^{+\infty}\left[\arctan \left(\frac{z+n z-x_{0}}{\Gamma}\right)-\arctan \left(\frac{z+n z+1-x_{0}}{\Gamma}\right)-\arctan \left(\frac{n z-x_{0}}{\Gamma}\right)+\arctan \left(\frac{n z+1-x_{0}}{\Gamma}\right)\right] .
\end{gathered}
$$

Examining the expression we can note that only the terms with $n=0$ survive in the sum but that terms simplify with the external factor (the constant $A$ ) so finally

$$
\int_{0}^{1} \rho(x, t) d x=1 \forall t
$$

Checking that Eq.(47) fulfils the Frobenius-Perron operator involves some extended but straightoforward algebra. 


\section{REFERENCES}

[1] A.N.Kolmogorov,Dokl.Acad.Sci.USSR 119(5), 861 (1958)

[2] Ya.G. Sinai,Dokl.Acad.Sci.USSR 124(4),768 (1959).

[3] W.H. Zurek, and J.P. Paz, Phys.Rev.Lett. 72, 2508 (1994).

[4] V. Latora and M. Baranger, Phys. Rev.Lett. 82, 520 (1999).

[5] S. Goldstein and O. Penrose, J. Stat. Phys. 24, 325 (1981)

[6] T. Petrosky and I. Prigogine, Chaos, Soliton \& Fractals 7,441 (1996).

[7] T. Petrosky and I. Prigogine, Chaos, Soliton \& Fractals 11,373 (2000).

[8] C. Beck, F. Schlögl, Thermodynamics of chaotic systems, Cambridge University Press, Cambridge (1993).

[9] Ya.B. Pesin, Russian Mathematical Surveys (4)32,55(1977).

[10] J.L. Lebowitz, Physica A 263, 516 (1999).

[11] D.J. Driebe, Fully Chaotic Maps and Broken Time Symmetry, Kluwer Academic Publishers, Dordrecht, The Netherlands, 1999.

[12] A. Lasota, M.C. Mackey, Chaos, Fractals and Noise, Stochastic Aspects and Dynamics, Second Edition, Springer-Verlag, New York (1994), p.13.

[13] M.C.Mackey, Time's Arrow:The Origin of Thermodynamic Behavior, Springer-Verlag, New York (1992).

[14] J.R. Dorfman, An Introduction to Chaos in Nonequilibrium Statistical Mechanics, Cambridge University Press, Cambridge (1999).

[15] C. Tsallis, A.R. Plastino, and W.-M.Zheng, Chaos, Soliton, Fractals,8, 885 (1997).

[16] L. Tessieri, D. Vitali, P. Grigolini, Phys. Rev. A 51, 4404 (1995). 
[17] E. Floriani, R. Mannella, P. Grigolini, Phys. Rev. E 52, 5910 (1995).

[18] M. Bologna, P. Grigolini, and J. Riccardi, Phys.Rev. E 82 , 6432 (1999).

[19] R.F. Fox, Chaos 8, 462 (1998).

[20] M. Abramowitz and I.A. Stegun, Handbook of Mathematical Functions, Dover, New York (1972) p. 804.

[21] A. Pattanayak and P. Brumer, Phys. Rev. E 56, 5174 (1997).

[22] A. Pattanayak and P. Brumer, Phys. Rev. Lett. 79, 4131 (1997).

[23] G. M. Zaslavsky, Physics of Chaos in Hamiltonian Systemson the Foundations of Statistical Physics,Imperial College Press, London (1988).

[24] G.M. Zaslavsky, Physics Today 52(8), 39 (1999).

[25] M. Ignaccolo, P. Grigolini, in preparation for Phys. Rev. E.

[26] C. Tsallis, J. Stat. Phys.52, 479 (1988). 